\title{
Simulation of a CME-driven shock by anisotropic scattering angular distributions
}

\author{
Xin Wang ${ }^{1,2}$ and Yi-Hua Yan ${ }^{1}$ \\ ${ }^{1}$ Key laboratory of Solar Activities, National Astronomical Observatories, Chinese Academy of \\ Sciences, Beijing 100012, China; email: wangxin@nao.cas.cn; yyh@nao.cas.cn \\ ${ }^{2}$ State Key Laboratory of Space Weather, Chinese Academy of Sciences, Beijing 100080, China;

\begin{abstract}
Observations of the interplanetary shock provide us with strong evidences of particle acceleration to multi-MeV protons in a coronal mass ejection (CME). Diffusive shock acceleration (DSA) is an efficient mechanism for cosmic ray (CR). This work presents a dynamical Monte Carlo simulation of a CME-driven shock on 14-Dec-2006 by using a series of Gaussian scattering angular distributions. With the simulated results, we find that particle energy spectrum is affected by energy injection processes under the anisotropic scattering law.
\end{abstract}

Keywords. acceleration of particles, shock waves, Sun: coronal mass ejections (CMEs), solar wind, methods: numerical

The unusual group of CME-driven shock events of solar cycle 23 was observed in December 2006 at the solar active region 10930. As shown in the first plot of Figure 1, an overview of key parameter observations from the Proton Monitor (PM) instruments on Wind/SWE for the CME shock event of 14 December 2006 are given in detail (Rosenvinge et al. 2009). According to this SEP event, we performed a Monte Carlo model (Wang \& Yan 2011; Wang \& Yan 2012) to investigate shock structure, particle injection and energy spectrum of the CME-driven shock. We consider a plane-parallel shock which was observed by Wind spacecraft in the location of the first Lagrangian point $L 1 \sim 1.5$ million $\mathrm{km}$ upstream of the Earth on 14 December. The important physical parameters of this simulation include the upstream bulk flow velocity $\left(u_{u}=-600 \mathrm{~km} / \mathrm{s}\right)$, the downstream bulk flow velocity $\left(u_{d}=-1042 \mathrm{~km} / \mathrm{s}\right)$, the relative bulk inflow velocity difference $(\Delta u=442 \mathrm{~km} / \mathrm{s})$, the upstream thermal velocity $\left(v_{0}=46 \mathrm{~km} / \mathrm{s}\right)$, and the constant scattering time $(\tau=0.052 \mathrm{~s})$. The numerical parameters include the box size $\left(x_{\max }=10 R_{e}\right)$, the time to evolve the whole system $\left(t_{\max }=6.3^{\prime}\right)$, the number of grid zones $\left(n_{x}=600\right)$, the initial number of particles per zone $\left(n_{0}=650\right)$, and the size of the time step $(d t=0.0053 \mathrm{~s})$. The presented simulations apply the same steps like the Earth-bow shock model (Knerr, Jokipii, \& Ellison 1996) including three sub steps: (i) All the particles moving with their velocities in the simulation box along the $x$ axis direction. (ii) Summing particle masses and velocities over each background computational grid. (iii) Invoking the scattering angular distribution law. The variation in the pitch angle $\delta \theta$ and azimuthal angle $\delta \phi$ are composed of the scattering angle, and its anisotropic character is described by the Gaussian function $f(\delta \theta, \delta \phi)$ in four cases with series of standard deviation value $\sigma=\pi / 4$ in Case A, $\sigma=\pi / 2$ in Case B, $\sigma=\pi$ in Case C, and isotropy in Case D. The simulated results of the bulk flow velocity, thermal velocity and proton density profiles in Case $\mathrm{C}$ are shown in the second plot of Figure 1.

As shown in the left plot of Figure 2, the fluency spectra of the proton in the 14-Dec2006 shock with spectral index 1.07 in the lower energy range are measured by multiple spacecraft. Simultaneously, as shown in the right plot of Figure 2, the four energy spectra with their indices of $\Gamma_{a}=1.3454$ in Case A, $\Gamma_{b}=1.2120$ in Case B, $\Gamma_{c}=1.1074$ in Case 

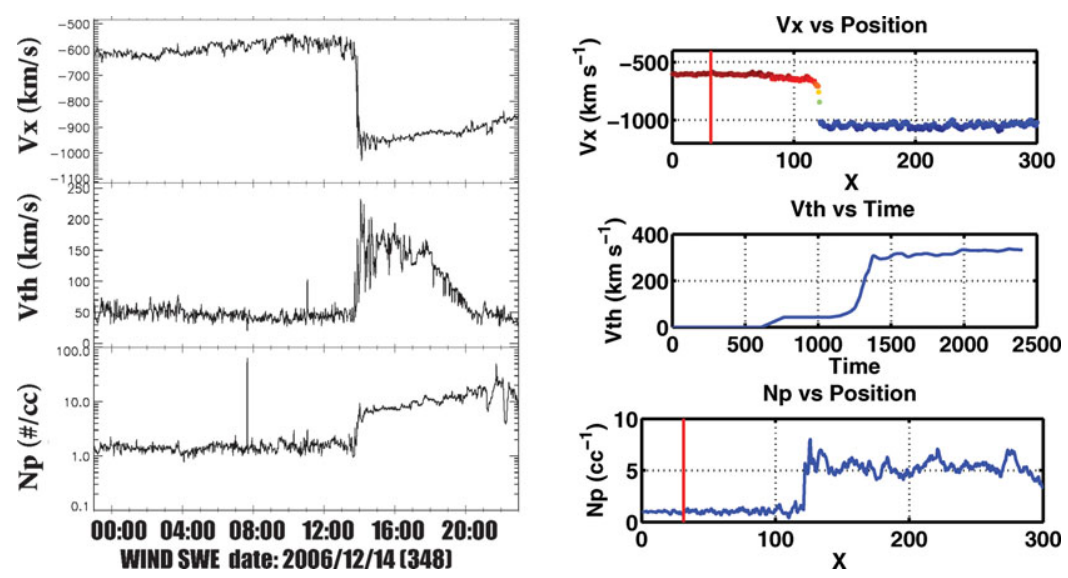

Figure 1. The first plot shows the key parameters of the 14-Dec-06 shock event in the Wind spacecraft, and the data come from http://cdaweb.gsfc.nasa.gov/cdaweb. The second plot shows the simulated results of the bulk flow speed profile, thermal velocity profile and proton density profile in Case $\mathrm{C}(\sigma=\pi)$.
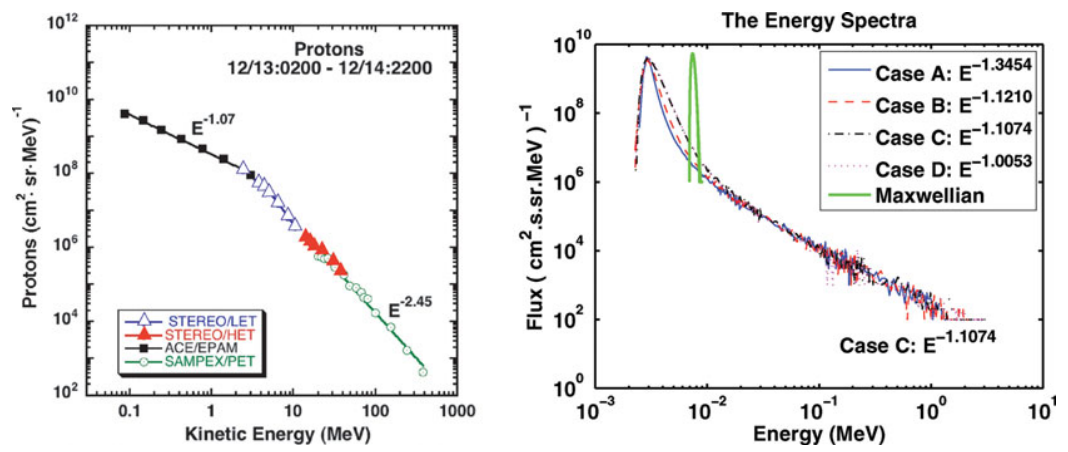

Figure 2. Fluency spectra of the protons measured in December 2006 SEP events by multiple spacecraft (Mewaldt et al. 2008). The right plot shows the simulated energy spectra on the energy range below $10 \mathrm{MeV}$ in four cases.

$\mathrm{C}$, and $\Gamma_{d}=1.0053$ in Case D are obtained. The energy spectral index of Case C provided a good fit to the observation in the lower energy range.

In conclusion, the dynamical Monte Carlo simulation of the 14-Dec-2006 CME-driven shock demonstrates that the energy spectrum is affected by the energy injection processes under the anisotropic scattering angular distributions. We can understand how the CMEdriven shock distributes its shock energy to accelerate the energetic particles by first-order Fermi acceleration mechanism and how it heats the background bulk flow at a certain efficiency.

\section{References}

Knerr, J. M., Jokipii, J. R., \& Ellison, D. C. 1996, ApJ 458, 641

Mewaldt, R. A. et al. 2008, in: R. Caballero et al. eds., SH, Proc. 30th ICRC (Mérida), 1, 107 Wang, X. \& Yan, Y. 2011, A\& $A, 530,92$

Wang, X. \& Yan, Y. 2012, Research in Astro. 83 Astrophys. 12, 1535-1548

von Rosenvinge T. T., Richardson, I. G., Reames, D. V., et al. 2009, Solar Phys. 256,443-462 